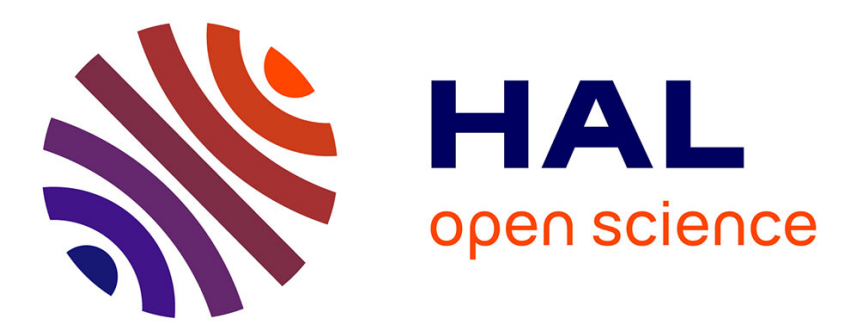

\title{
A dimensional analysis of forest fuel layer ignition model: Application to the ignition of pine needle litters
}

Aymeric Lamorlette, Mohamad El Houssami, Jan C. Thomas, Albert Simeoni, Dominique Morvan

\section{- To cite this version:}

Aymeric Lamorlette, Mohamad El Houssami, Jan C. Thomas, Albert Simeoni, Dominique Morvan. A dimensional analysis of forest fuel layer ignition model: Application to the ignition of pine needle litters. Journal of Fire Sciences, 2015, pp.NC. 10.1177/ToBeAssigned . hal-01157866

\section{HAL Id: hal-01157866 https://hal.science/hal-01157866}

Submitted on 1 Jun 2015

HAL is a multi-disciplinary open access archive for the deposit and dissemination of scientific research documents, whether they are published or not. The documents may come from teaching and research institutions in France or abroad, or from public or private research centers.
L'archive ouverte pluridisciplinaire HAL, est destinée au dépôt et à la diffusion de documents scientifiques de niveau recherche, publiés ou non, émanant des établissements d'enseignement et de recherche français ou étrangers, des laboratoires publics ou privés. 


\title{
A dimensional analysis of forest fuel layer ignition model: Application to the ignition of pine needle litters
}

\author{
Aymeric Lamorlette ${ }^{1}$, Mohamad El Houssami ${ }^{2}$, Jan C. Thomas ${ }^{2}$, \\ Albert Simeoni $^{2}$ and Dominique Morvan ${ }^{1}$
}

\begin{abstract}
This paper deals with the physical modelling of forest fuel layer ignition. A model based on momentum, fluid and solid phase energy equations is written for a fuel layer and a dimensional analysis is performed. This analysis allows to enlighten two relevant dimensionless groups regarding the dimensionless time to ignition of a fuel layer and also provides a suited scaling for the fluid velocity inside the fuel layer during ignition. A correlation for the time to ignition is then fitted on experimental data obtained using a FM-Global Fire Propagation Apparatus (FPA) for different pine species with a closed basket. A good agreement is found, emphasizing the relevance of the dimensionless groups and the thermally thick behaviour of the solid particles during the ignition process under incident radiant heat flux as low as $8-12 \mathrm{~kW} \cdot \mathrm{m}^{-2}$.
\end{abstract}

keywords: Forest fuel layer ignition, dimensional analysis, thermal behaviour, ignition time correlations

\section{Introduction}

Ignition of solid material exposed to radiant heat fluxes is a topic that has been widely investigated in the case of optically thick materials, providing the "classical ignition theory" [1]. This theory considers that the radiant heat flux is fully absorbed by the solid material surface, which ignites when

\footnotetext{
${ }^{1}$ Aix Marseille Université, CNRS, Centrale Marseille, M2P2 UMR 7340, 13451 Marseille, France

${ }^{2}$ BRE Centre for Fire Safety Engineering, The University of Edinburgh, United Kingdom

Corresponding author: aymeric.lamorlette@univ-amu.fr
} 
the surface temperature reaches a given ignition temperature $T_{s, i g}$, neglecting any mixing or chemical time. These assumptions can be considered as rather crude. According to experimental observations, ignition temperature depends on the imposed heat flux, as chemical and mixing times. Nevertheless, these approximations seem acceptable for fire safety applications beyond the critical heat flux [1]. The total heat flux at the solid surface is estimated as the sum of the net radiant heat flux and a total equivalent convective heat flux. The square root of time to ignition $t_{i g}$ is then proportional to the inverse of the incident radiant heat flux $\Phi_{0}^{\prime \prime}$ in the case of high fluxes (beyond the critical flux) and thermally thick material:

$$
\frac{1}{\sqrt{t_{i g}}}=\frac{2}{\sqrt{\pi} \sqrt{\lambda_{s} \rho_{s} C p_{s}}} \frac{\epsilon \Phi_{0}^{\prime \prime}}{\left(T_{s, i g}-T_{0}\right)}
$$

Where $\lambda_{s}$ is the solid conductivity, $\rho_{s}$ the solid density, $C p_{s}$ the solid specific heat, $\epsilon$ the solid particle emissivity and $T_{0}$ the ambient temperature.

However, when the incident heat flux reaches important values (above $70 \mathrm{~kW} \cdot \mathrm{m}^{-2}$ ), divergence from this theory is observed [2,3], showing a time to ignition higher than the predicted values. This behaviour has been theoretically explained using an in-depth radiation assumption in the material $[4,5]$.

Adaptation of this theory to forest fuel layers has been proposed [6, 7], where either an experimental fitting of the total heat transfer coefficient had to be done [6], or a prescribed constant value which maximise the heat transfer had to be used [7] for this coefficient: The solid surface flux composed of a radiant and a convective term can be well estimated for classical solid material, whereas it seems harder when applied to forest fuel layers. Indeed, the convective heat transfer within the fuel layer is driven by an induced momentum transfer in no-wind conditions [8] that needs to be firstly evaluated. Hence, in order to accurately take into account the convective heat transfer, a model suited for forest fuel layers must be used. The radiation absorption coefficient of a fuel layer can be estimated using the classical DeMestre relation $[9,10]$ or using direction-dependent relation [11]. Therefore, transposition of the in-depth radiation concept to forest fuel layer could be proposed even at low heat fluxes since the radiation absorption coefficient of a fuel layer is often more than ten times smaller than black PMMA absorption coefficient, for which a surface absorption of the radiant heat flux can be considered.

This is why, in order to account for the particular behaviour of forest fuel layers, the ignition model must be able to represent both in-depth radiation and natural convection caused by induced momentum. Besides, the question of the needle thermal behaviour is of prior interest to model the fuel layer behaviour. Indeed, assuming a thermally thin behaviour suggests to model the fuel layer using an equivalent medium, whereas assuming a thermally thick behaviour would require to model specifically the particles. According 
to a study on the thermal behaviour limit [12], these two approaches are acceptable, given the particle size and the commonly imposed heat fluxes. Nevertheless, it seems, according to the same study [12], that the needle thermal behaviour is thermally thick in most ignition cases. This result is in accordance with experimental measurements $[6,7]$, which show that the inverse of the square root of the time to ignition still seems to depend linearly on the imposed radiant heat flux. Hence, the model equations will not consider an equivalent medium for the fuel layer as often proposed for ignition modelling [7, 13] or for forest fire propagation study [14, 15, 16]. A classical model is chosen instead as it can be suggested for the solid phase [17]. Moreover, the equivalent medium equation considers an average solid temperature whereas the ignition process seems to depend on a surface temperature regarding the classical ignition theory. These two models lead to different expressions of the characteristic time and the equivalent medium characteristic time is not consistent with experimental observations [7, 13]. Thus, the characteristic time for the solid material, defined as an effusion time is:

$$
t_{r e f}=\frac{\pi}{4} \lambda_{s} \rho_{s} C p_{s}\left(\frac{T_{s, i g}-T_{0}}{\epsilon \Phi_{0}^{\prime \prime}}\right)^{2}
$$

Finally, this model allows characterising the solid particle behaviour during the ignition process rather than the behaviour of the equivalent medium as in previous experimental studies [7]. It also helps distinguishing the role of the solid particles from the role of the packing in the fuel layer ignition process. Indeed, according to the theoretical thermal limit, this reference time is more suited to represent the behaviour of sole pine needles [12], if considering heat flux ranges used in previous experimental studies $[7,13]$ for instance.

The paper is organized as follow: In order to develop a dimensional model for ignition time of forest fuel layers, the studied system equations are presented along with the characteristic scales used to write them in a dimensionless form. The role of the different dimensionless groups appearing in these equations is then discussed and a suited scaling is defined for the fluid velocity inside the fuel layer during ignition. Several experimental data on radiation induced piloted ignition, involving numerous pine species, fuel layer packings and heat fluxes, are then used to evaluate the relevance of the different dimensionless numbers. This investigation is performed for a standard experimental configuration, using a FM-Global Fire Propagation Apparatus (FPA) [18]. Then, a simple correlation is provided for forest fuel layer ignition time that is accurate on a broad range of experimental conditions. 


\section{Physical modelling}

The system studied here, presented in Fig. 1 is composed of a fuel layer exposed to an incident radiant heat flux heating the solid. The highest solid temperature, expected at the fuel layer surface, is responsible for ignition. However, an in-depth radiation increases the solid temperature within the fuel layer. The temperature difference between the solid and the fluid within the layer is responsible for a convective heat transfer that generates an induced momentum. This induced momentum is then enhancing the convective heat transfer at the layer surface, modifying the heat transfer and the time to ignition. Thus, equations must model the momentum transfer in the fluid phase, the heat transfer in both phases as well as in-depth radiation.

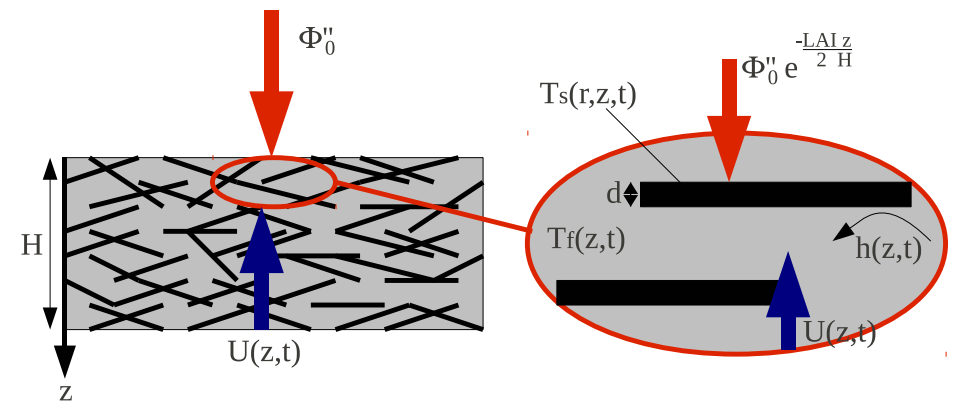

Figure 1: Sketch of the ignition problem

\subsection{Set of equations}

The following equation is proposed for the momentum modelling, considering a buoyant and a drag term due to fluid-vegetation interactions:

$$
\frac{\partial u}{\partial t}+(u . \nabla) u=-\frac{1}{\rho_{f}} \nabla p+\nu_{f} \Delta u+g \frac{T_{f}-T_{0}}{T_{f}}-C d \frac{\alpha_{s} \sigma_{s}}{2}|u| u
$$

Where $u$ is the fluid velocity, $p$ the total pressure, $\rho_{f}$ the fluid density evaluated at $T_{f}, \nu_{f}$ the fluid viscosity, $g$ the gravity acceleration, $T_{f}$ the fluid temperature, $C d$ the drag coefficient of vegetation usually set [19] at $C d=0.15$. This coefficient can be more accurately evaluated for needle litters [20]. For the packing ratios and specific areas considered in this study, it seems nevertheless acceptable to consider a constant value. $\alpha_{s}$ is the solid fraction and $\sigma_{s}$ the specific area of vegetation. The additional term due to density variation coming from the divergence of the strain rate tensor is here neglected. 
Using a low Mach number approximation, this term depends straightforwardly on the right hand side of the fluid phase energy equation. Thus, the dimensionless groups driving the system remain the same.

The following equation is proposed for the fluid phase energy model, considering the fluid temperature around a solid particle and a convective heat transfer between the solid particles and the fluid phase:

$$
\rho_{f} C p_{f}\left(\frac{\partial T_{f}}{\partial t}+u . \nabla T_{f}\right)=\lambda_{f} \Delta T_{f}+\chi\left(T_{s, \text { surf }}-T_{f}\right)
$$

Where $C p_{f}$ the fluid specific heat and $\chi=h \alpha_{s} \sigma_{s}$ the heat transfer coefficient $[8,21], h$ being the "classical" convective heat transfer coefficient.

Finally, the following equation is proposed for a solid particle, neglecting the fuel moisture content (FMC):

$$
\rho_{s} C p_{s} \frac{\partial T_{s}}{\partial t}=\lambda_{s} \Delta T_{s}
$$

The boundary condition at the surface of the solid is:

$$
-\lambda_{s} \nabla_{\text {surf }} T_{s}=\epsilon \Phi_{\text {net }}^{\prime \prime}-h\left(T_{s, \text { surf }}-T_{f}\right)
$$

Where $\Phi_{\text {net }}^{\prime \prime}$ is the net radiant heat flux depending on both solid surface temperature and radiation extinction within the fuel layer. The surface temperature dependency of $\Phi_{\text {net }}^{\prime \prime}$ will here be neglected, assuming that the radiation re-emitted by the surface is small compared to $\Phi_{0}^{\prime \prime}$ during the ignition process. This assumption is questionable but does not affect the following dimensional analysis. This radiant heat flux is then depending on the extinction through the fuel layer which is the exponential of the fuel layer optical thickness $\frac{L A I}{2}=H \frac{\alpha_{s} \sigma_{s}}{4}$ where $H$ is the fuel layer height and $L A I$ the leaf area index [22]. However, as a first approximation, $\Phi_{\text {net }}^{\prime \prime}$ will be considered equal to $\Phi_{0}^{\prime \prime}$, taking into account the in-depth radiation via $L A I$. This is a rather crude assumption as reflection can occur in the litter. Also, variations of the extinction coefficient can occur due to specific pine needle orientation. Nevertheless, these phenomena are expected not to be too different from a pine litter to another. Hence this assumption should be valid for needle litter, but it could differ for other kind of vegetations such as leaf litters for instance.

\subsection{Characteristic time and length scales}

The system exhibits two length scales: One at the scale of the particles in the system (i.e. at the needle scale) and one at the layer scale (i.e. at the bulk scale). A subscript $\mathrm{p}$ is associated to the particle scale and a subscript $\mathrm{b}$ is associated to the layer scale. Two characteristic time scales have to be defined: The first one $\left(t_{r e f, \mathrm{p}}=t_{r e f}\right)$ at particle scale is related to the heat 
transfer ; the second one $\left(t_{r e f, \mathrm{~b}}\right)$ is related to the momentum transfer at the fuel layer scale. $t_{r e f, \mathrm{~b}}$ can then be defined as $t_{r e f, \mathrm{~b}}=H / U_{\text {ref }}$ where $U_{r e f}$ is a suited velocity scale. The use of the following velocity scale is proposed based on buoyant forces $U_{r e f}=\sqrt{2 g H\left(T_{i g}-T_{0}\right) / T_{i g}}$, noticing that another velocity scale considering equilibrium between buoyant and drag forces could also be defined: $U_{\text {lim }}=\sqrt{2 g\left(T_{i g}-T_{0}\right) /\left(C d \alpha_{s} \sigma_{s} T_{i g}\right)}$. The relevance of these characteristic velocities will be discussed in a following section.

A velocity and a length scale have been defined at bulk scale, a length scale is now defined at particle scale. Considering a fuel layer composed of cylinders (this assumption seems relevant for needle litters [23]), the needle diameter can be defined as $d=4 / \sigma_{s}$, allowing the definition of the particle length scale.

\subsection{Dimensionless equations}

\subsection{1 momentum equation}

The following set of dimensionless variables is proposed: $T_{\mathrm{b}}^{*}=\left(T_{f}-T_{0}\right) / T_{f}$, $z_{\mathrm{b}}^{*}=z / H, u^{*}=u / U_{r e f}, t_{\mathrm{b}}^{*}=t / t_{r e f}$, b with $z$ being a bulk spatial coordinate. Using a classical methodology [24] leads to:

$$
\frac{\partial u^{*}}{\partial t_{\mathrm{b}}^{*}}+\left(u^{*} \cdot \nabla_{\mathrm{b}}^{*}\right) u^{*}=-\nabla_{\mathrm{b}}^{*} p^{*}+\frac{1}{R e_{\mathrm{b}}} \Delta_{\mathrm{b}}^{*} u^{*}+\frac{k}{2} g^{*}-C d L A I\left|u^{*}\right| u^{*}
$$

Where $k=\frac{T_{i g}}{T_{i g}-T_{0}} T_{\mathrm{b}}^{*}, g^{*}=\frac{g}{\|g\|}, L A I=\frac{\alpha_{s} \sigma_{s}}{2} H$ and $R e_{\mathrm{b}}=\frac{H U_{r e f}}{\nu_{f}}$ is the bulk Reynolds number. Introducing the definition of the reference velocity in the bulk Reynolds number leads to $R e_{\mathrm{b}}^{2}=\frac{2 H^{3} g\left(T_{i g}-T_{0}\right)}{\nu_{f}^{2} T_{i g}}$. Hence $R e_{\mathrm{b}}^{-1}$ can be neglected (and then the diffusion term) when $H \geq\left(\frac{\nu_{f}^{2} T_{i g}}{2\left(T_{i g}-T_{0}\right) g \epsilon_{R e}^{2}}\right)^{1 / 3}$ where $\epsilon_{R e}$ is the threshold beyond which $R e_{\mathrm{b}}^{-1}$ cannot be neglected. This threshold can be evaluated based on the fact that the order of the other terms in this equation is unity, suggesting to set $\epsilon_{R e}=10^{-2}$ for instance. In that case, the diffusion term can be considered as negligible as long as $H \geq 510^{-3} \mathrm{~m}$, which is much smaller than the typical height of forest fuel layers. Hence it will always be neglected during the ignition process, leading to re-write Eq. (7):

$$
\frac{\partial u^{*}}{\partial t_{\mathrm{b}}^{*}}+\left(u^{*} \cdot \nabla_{\mathrm{b}}^{*}\right) u^{*}=-\nabla_{\mathrm{b}}^{*} p^{*}+\frac{k}{2} g^{*}-C d L A I\left|u^{*}\right| u^{*}
$$

Since $t_{r e f, \mathrm{~b}}<<t_{r e f, \mathrm{p}}$ for most fuel layers (in this study the ratio $t_{r e f, \mathrm{~b}} / t_{r e f, \mathrm{p}}$ is always lower than $1 \%$, according to Table 2$)$, a quasi-static assumption, i.e. considering a slow temperature evolution regarding the fluid motion evolution, can also be proposed to define the reference velocity at a given temperature. The definition of such reference velocity is necessary to estimate the convective heat transfer in the fuel layer $[8,21]$. Indeed, 
the Nusselt number that accounts for convective heat transfer is temperature dependent, whereas the reference velocity $U_{\text {ref }}$ is evaluated at ignition temperature. Hence a velocity evaluated at the medium instantaneous temperature has to be defined in order to evaluate the Nusselt number. This is the reason why another temperature dependent reference velocity is defined. Eq (3) can then be made dimensionless (making the same assumption on the bulk Reynolds number) using the following reference velocity $U_{\text {ref, } 2}=\sqrt{2 g H\left(T_{f}-T_{0}\right) / T_{f}}$ :

$$
\frac{\partial u^{*}}{\partial t_{\mathrm{b}}^{*}}+\left(u^{*} \cdot \nabla_{\mathrm{b}}^{*}\right) u^{*}=-\nabla_{\mathrm{b}}^{*} p^{*}+\frac{1}{2} g^{*}-C d L A I\left|u^{*}\right| u^{*}
$$

\subsection{2 solid phase energy equation}

The following set of dimensionless variables is proposed: $T_{s, \mathrm{p}}^{*}=T_{s} / T_{0}$, $r_{\mathrm{p}}^{*}=r \sigma_{s} / 4, t_{\mathrm{p}}^{*}=t / t_{r e f, \mathrm{p}}$ where $r$ is a particle spatial coordinate. This leads to re-write Eq. (5) as:

$$
16 B i^{2} \frac{\partial T_{s, \mathrm{p}}^{*}}{\partial t_{\mathrm{p}}^{*}}=\Delta_{\mathrm{p}}^{*} T_{s, \mathrm{p}}^{*}
$$

Where $B i=\frac{\epsilon \Phi_{0}^{\prime \prime}}{\lambda_{s} \sigma_{s}\left(T_{s, i g}-T_{0}\right)}$ is a radiative Biot number [25].

\subsection{3 solid phase energy equation boundary condition}

The following dimensionless variable is proposed for the fluid temperature: $T_{f, \mathrm{~b}}^{*}=T_{f} / T_{0}$. Writing the Nusselt number:

$$
N u=\frac{h d}{\lambda_{f}}
$$

provides

$$
-\nabla_{\mathrm{p}, \operatorname{surf} f}^{*} T_{s, \mathrm{p}}^{*}=4 B i \frac{\left(T_{s, i g}-T_{0}\right)}{T_{0}}-\frac{\lambda_{f}}{\lambda_{s}} N u\left(T_{s, \mathrm{p}}^{*}\left(r^{*}=1 / 2\right)-T_{f, \mathrm{~b}}^{*}\right)
$$

Where the Nusselt number is depending on both Prandtl $(\operatorname{Pr})$ and particle Reynolds number. The following quasi-static Reynolds number, defined using characteristic velocity $U_{\text {lim, } 2}=\sqrt{2 g\left(T_{f}-T_{0}\right) /\left(C d \alpha_{s} \sigma_{s} T_{f}\right)}$ as discussed later in the article, is suggested: $R e_{\sigma_{s}}=U_{\text {lim, } 2} / \nu_{f} \sigma_{s}$.

\subsection{4 fluid phase energy equation}

The fluid phase energy equation shows two characteristic times or velocity scales. Indeed, the fluid temperature time evolution, mostly driven by the convective heat transfer at short time scales, should depend on the particle 
time scale, whereas the temperature gradient across the fuel layer should depend on the bulk velocity/time scale. This is why both scales should appear in this equation. However, this equation is scaled using the bulk scales to make the comparison between the different terms possible:

$$
\frac{\partial T_{f, \mathrm{~b}}^{*}}{\partial t_{\mathrm{b}}^{*}}+u^{*} \cdot \nabla_{\mathrm{b}}^{*} T_{f, \mathrm{~b}}^{*}=\frac{1}{\operatorname{Pr} R e_{\mathrm{b}}}\left(\Delta_{\mathrm{b}}^{*} T_{f, \mathrm{~b}}^{*}+\frac{N u L A I^{2}}{\alpha_{s}}\left(T_{s, \mathrm{p}}^{*}\left(r^{*}=1 / 2\right)-T_{f, \mathrm{~b}}^{*}\right)\right)
$$

The first term on the right-hand side of Eq. (13) can be neglected since the second term is much greater, except at very short time scales where $N u \sim 0$. Moreover, noticing that $\frac{L A I^{2}}{R e_{\mathrm{b}} \alpha_{s}}=\frac{L A I}{R e_{\sigma_{s}}} \sqrt{\frac{k}{C d L A I}}$ leads to:

$$
\frac{\partial T_{f, \mathrm{~b}}^{*}}{\partial t_{\mathrm{b}}^{*}}+u^{*} \cdot \nabla_{\mathrm{b}}^{*} T_{f, \mathrm{~b}}^{*}=\frac{N u L A I}{\operatorname{Pr} R e_{\sigma_{s}}} \sqrt{\frac{k}{C d L A I}}\left(T_{s, \mathrm{p}}^{*}\left(r^{*}=1 / 2\right)-T_{f, \mathrm{~b}}^{*}\right)
$$

Thermo-physical properties of pine species used along this study are from various studies $[26,27,28,29]$ in addition to new measurements. These parameters are listed in Table 1. The thermo-physical properties of the fluid are those of air as a first approximation, since the surrounding evolves from air to pyrolysates during ignition. Nevertheless, since thermal degradation is about the same for every pine needle species, these properties can be roughly considered as constant from one ignition to another and also can be roughly considered as being those of air. Emissivity values are related to mean values, using a Planck averaging for wood on wavelengths emitted by FPA lamps [30]. Therefore, it should be noted that comparison with data obtained using other devices should use different values for the emissivity.

\begin{tabular}{|c||c|c|c|}
\hline Species & $\epsilon[-]$ & $T_{s, i g}[\mathrm{~K}]$ & $\lambda\left[\mathrm{W} . \mathrm{m}^{-1} . \mathrm{K}^{-1}\right]$ \\
\hline \hline Pinus Halepensis (PH) & 0.6 & 600 & 0.12 \\
\hline Pinus Strobus (PS) & 0.6 & 600 & 0.12 \\
\hline Pinus Pinaster (PM) & 0.6 & 600 & 0.12 \\
\hline \hline Species & $\rho\left[\mathrm{kg} . \mathrm{m}^{-3}\right]$ & $C p\left[{\left.\mathrm{~J} . \mathrm{kg}^{-1} . \mathrm{K}^{-1}\right]}_{1}\right.$ & $\sigma_{s}\left[\mathrm{~m}^{-1}\right]$ \\
\hline Pinus Halepensis (PH) & 789 & 1827 & 7377 \\
\hline Pinus Strobus (PS) & 621 & 2400 & 8183 \\
\hline Pinus Pinaster (PM) & 927 & 2017 & 5500 \\
\hline
\end{tabular}

Table 1: Solid particles physical properties [26, 27, 28, 29, 30], plus some from this study

\section{The different dimensionless groups and their rel- evance}

The following dimensionless groups have been identified in the dimensionless system of equations: $C d L A I, \frac{\left(T_{i g}-T_{0}\right)}{T_{i g}}, B i, \frac{\left(T_{s, i g}-T_{0}\right)}{T_{0}}, \frac{\lambda_{f}}{\lambda_{s}}, \operatorname{Re} e_{\sigma_{s}}$ and $\operatorname{Pr}$ (via $N u$ ) and finally $L A I$ to take into account in-depth radiation. 


\subsection{Definition of a relevant velocity scale}

It can be noticed that:

$$
\frac{U_{\text {ref }}}{U_{\text {lim }}}=\sqrt{2 C d L A I}
$$

Therefore the choice of the characteristic velocity does not affect the dimensional analysis since the velocity ratio is only depending on $C d L A I$ which is one of the dimensionless groups involved in this study. However, the definition of a physically meaningful velocity scale is an interesting question regarding the model range of validity. Taking Eq. (8), setting $C d L A I<<1$ provides a standard buoyant flow, so that $U_{r e f}$ is a suited velocity scale. For $C d L A I>>1$, the drag force dominates in order to reach the limit velocity quickly. Then $U_{\text {lim }}$ should be used as the velocity scale. A rough approximation of the characteristic velocity transition is given by Eq. (15) at $C d L A I=0.5$. Since most forest fuel layers as needles [26] or shrubs [31] exhibit $C d L A I>0.5, U_{\text {lim }}$ is chosen as velocity scale.

A more detailed study can be provided solving Eq. (9) without pressure gradient in one dimension with $u(t=0)=0$. The solution is then evaluated at the time $\tau_{\text {trav }}$ needed to travel through the layer and compared to the reference buoyant velocity $U_{r e f, 2}$. Both solutions are made dimensionless using the limit velocity $U_{l i m, 2}$, providing:

$$
\frac{u\left(\tau_{\text {trav }}\right)}{U_{\text {lim }}}=\tanh (\operatorname{Argch}(\exp (C d L A I)))
$$

These curves are presented in Fig. 2, emphasizing that the limit from buoyant to limit flow is located at $C d L A I=0.5$ with a pure buoyant flow at $C d L A I<<1$ and a pure limit flow at $C d L A I>>1$. The difference between the solution of Eq. (8) and Eq. (9) has to be discussed to apply this remark in the case of a non quasi-static evolution. Thanks to the behaviour of $k$ at short time scale, Eq. (8) indicates that the solution Eq. (16) should be shifted to the left (toward small values of $C d L A I$ ), making the curve stiffer while solution Eq. (16) is recovered at ignition since $k$ tends toward 1 when $T_{f}$ tends toward $T_{i g}$. This remark emphasizes the fact that the flow during the ignition process is closer to the limit flow.

Because of the previous remark on fuel layers values of the $C d L A I$ parameter, the particle Reynolds number is then defined using $U_{l i m, 2}$ rather than $U_{r e f, 2}$.

\subsection{Assumption on the dimensionless groups}

To develop a practical ignition time correlation applicable for fire safety, the following assumptions are suggested to reduce the amount of dimensionless groups involved in the relation. Hence, to get a simple expression for the ignition time with an acceptable accuracy, some strong assumptions are suggested, and discussed after fitting the correlation. 


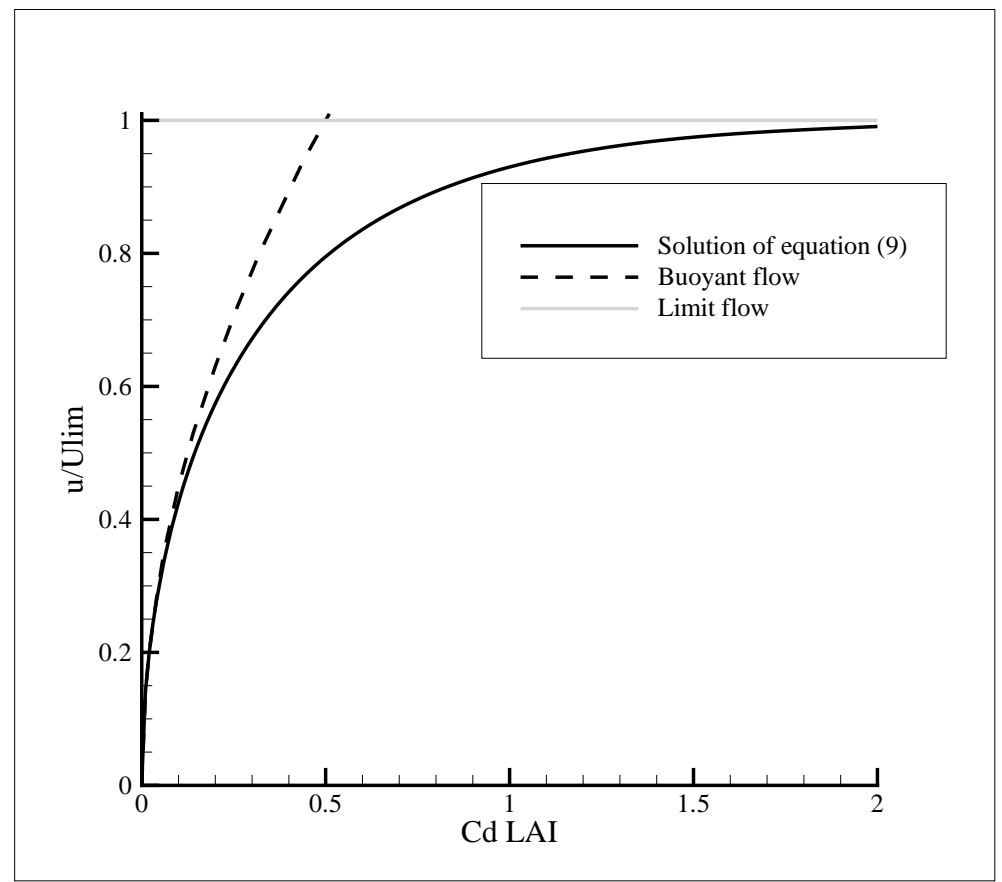

Figure 2: Flow behaviour regarding $C d L A I$

- The fluid surrounding the fuel, thus its physical properties, is considered to remain constant before ignition. This assumption seems acceptable since ignition is always occurring with air as surrounding medium. As the solid is considered as inert until ignition, the gas phase composition does not change much before ignition. Close to ignition, pyrolysates are appearing in the surrounding but the composition change could be considered as identical from one ignition situation to another (when considering pine needle litters), as suggested earlier. Hence, considering constant properties, evaluated at a mean temperature between ambient and ignition temperature, could be reliable.

- The relative variations of the particles physical properties $\left(\lambda_{s}, \rho_{s}, C p_{s}\right.$, $\left.T_{s, i g}\right)$ can be considered small compared to bulk properties relative variations $\left(\alpha_{s}, H\right)$ in the dimensionless groups, according to the parameter ranges provided in various studies [7, 13, 26, 27, 28, 29].

- The drag coefficient $C d$ is considered as constant so that $C d L A I$ and $L A I$ are equivalent dimensionless groups. Indeed, this assumption is almost always true [20] for fuel layers of interest [7, 13]. Besides, according to the solid fraction and specific area of pine needles considered in this study, the relative variation of $C d$ can be considered as 
negligible compared to the relative variation of $L A I$.

On account of the two first assumptions, the following dimensionless groups can be discarded, despite the fact that they actually have a significant role in the physics of litter ignition: $\operatorname{Pr}, \frac{\left(T_{i g}-T_{0}\right)}{T_{i g}}, \frac{\left(T_{s, i g}-T_{0}\right)}{T_{0}}$ and $\frac{\lambda_{f}}{\lambda_{s}}$ and the following remark is noticed: The particle quasi-static Reynolds $R e_{\sigma_{s}}$ number which depends on $T_{\mathrm{b}}^{*}$ is similar for a dimensional analysis to $\Psi$ at given $T_{\mathrm{b}}^{*}$ and $C d L A I$, introducing $\Psi=\frac{\sigma_{s}^{2} \nu_{f}^{2}}{g H}$, an intrinsic parameter of the fuel layer accounting for the induced flow behaviour (with $R e_{\sigma_{s}}^{2}=\frac{1}{\Psi C d L A I} \frac{T_{f}-T_{0}}{T_{f}}$ ).

Based on these assumptions, the following dimensionless groups can be considered to be the most relevant for developing a correlation on pine needle litter ignition time:

- $B i$, a radiative Biot number, depending on the solid material and experimental conditions [25].

- $C d L A I$, accounting for both induced momentum and in-depth radiation effects. This is a property of both the solid material and the fuel layer.

- $\Psi$, accounting for the induced flow hydrodynamic effects. This is a property of both the solid material and the fuel layer.

Of course, a FMC dependency should be added in the case of non dried fuels.

\subsection{The relevant dimensionless groups and their physical meaning}

Remarking here that the modification of the ignition process compared to the classical ignition theory seems mostly due to the in-depth radiation, the convective heat transfer and the associated induced momentum, $C d L A I$ and $\Psi$ are likely to be the most relevant dimensionless groups.

Potential limits for the correlation are now drawn thanks to the definition of $C d L A I$ and $\Psi$ : As previously suggested, $C d L A I$ is responsible for a transition from pure buoyant flow to a limit flow at $C d L A I=0.5$. Moreover, a transition from a boundary to a mixing layer over vegetation canopies exists at $C d L A I=0.1$ [32], suggesting that a correlation should be fitted differently for $C d L A I<0.1$ (and $0.1<C d L A I<0.5$ ). This limit is close to the one provided by the optical thickness for standard $C d$ values $(C d L A I=0.3)$. Since most fuel layers show $C d L A I>0.5$, this remark concerns very few forest fuel layers.

The role of $\Psi$ might be more difficult to enlighten. Indeed, along with $T_{\mathrm{b}}^{*}$ and $C d L A I$ it defines the particle Reynolds number $R e_{\sigma_{s}}$. This Reynolds 
number is accounting for the flow transitions in the fuel layer. The threedimensional vortex shedding transition occurring at $R e_{d}>200$ (corresponding to $R e_{\sigma_{s}}>50$ ) can be responsible for a turbulent-like flow transition through vortex dislocations in the fuel layer [20,33]. This transition is responsible for important modifications of the fluxes between the solid particles and the fluid phase so that the temperature raise leading to ignition is different if it occurs before or after this transition. Thus authors propose to distinguish ignitions where the flow remains almost always laminar during the ignition process from ignitions where a turbulent-like transition quickly occur. Since ignition occurs at $\left(T_{f}-T_{0}\right) / T_{f} \sim 0.5$ (considering that $T_{s, i g} \sim T_{f, i g}$ ) [29], the transition $\Psi$ can be defined using $R e_{\sigma_{s}, t_{i g}}=\sqrt{\frac{1}{\Psi C d L A I} \frac{T_{i g}-T_{0}}{T_{i g}}}$ providing $(\Psi C d L A I)_{l i m}=210^{-4}$. Therefore two different fittings of the correlation could be expected regarding the value of $\Psi$. However, the flow occurring during the ignition process for $\Psi$ just above the limit is still essentially laminar so that the time to ignition is not much affected by the turbulent transition. Turbulent effects on the time to ignition should then appear in situations where the limit Reynolds number is quickly reached. Hence, the latter should be increased of at least one order of magnitude to correctly estimate the transition. This leads to $(\Psi C d L A I)_{l i m}=210^{-6}$. Since every litters [26] and most shrubs [31] show $\Psi C d L A I \geq 210^{-4}$ and since every fuel layers show $\Psi C d L A I \geq 210^{-6}$, most fuel layers ignite before or just after the pseudo-turbulent transition. Therefore, this limit seems also negligible in most situations.

\section{A correlation for the time to ignition}

A correlation for time to ignition can now be proposed in the following form:

$$
t_{i g}^{*}=\frac{t_{i g}}{t_{r e f, \mathrm{p}}}=F(C d L A I, \Psi, B i)
$$

\subsection{Experimental protocol}

The experiments presented in this paper were conducted using a FPA, presented in Fig. 3. The FPA allows natural convection or forced gas flow rate through the fuel bed. However this study is focused on natural conditions therefore experiments were conducted only with natural convection. Specific sample holders were used for the experiments. They consisted in circular baskets, made of stainless steel. The studied fuel was dead and not conditioned Pinus Halepensis (PH) and Pinus Strobus (PS) needles. The FMC has been determined by oven drying samples for 24 hours at $60^{\circ} \mathrm{C}$. The fuel sample FMC ranged between $4.9 \%$ and $6.4 \%$. Baskets were filled up to the top with varying fuel mass load. Experimental results that have been used 
here are from various studies $[6,13,18]$, plus some original to this study. Experiments conducted on Pinus Pinaster [13] (PM for Maritime Pine) at various mass loads correspond to $\alpha_{s}$ in the range $[0.02 ; 0.08]$. Note that despite many other studies propose experimental ignition time for such material, these studies are not considered here to avoid any device dependency effects. Indeed, as previously explained, emissivity values considered in this study are related to wood under FPA lamps. Hence, if focused on needles under a FPA, only few experimental studies exist.

The following experimental conditions were used:

- The basket opening was $0 \%$.

- The mass load was 10 or $15 \mathrm{~g}$ with associated $\alpha_{s}$ equal to $3.74 \%$ and $5.6 \%$ for Pinus Halepensis. The mass load was either 10, 15 or $20 \mathrm{~g}$ with associated $\alpha_{s}$ equal to $4.75 \%, 7.12 \%$ and $9.5 \%$ for Pinus Strobus.

- The radiant heat flux varied from 8 to $55 \mathrm{~kW} / \mathrm{m}^{2}$.

Ignition time is identified as the time where the sharpest mass loss evolution is observed.

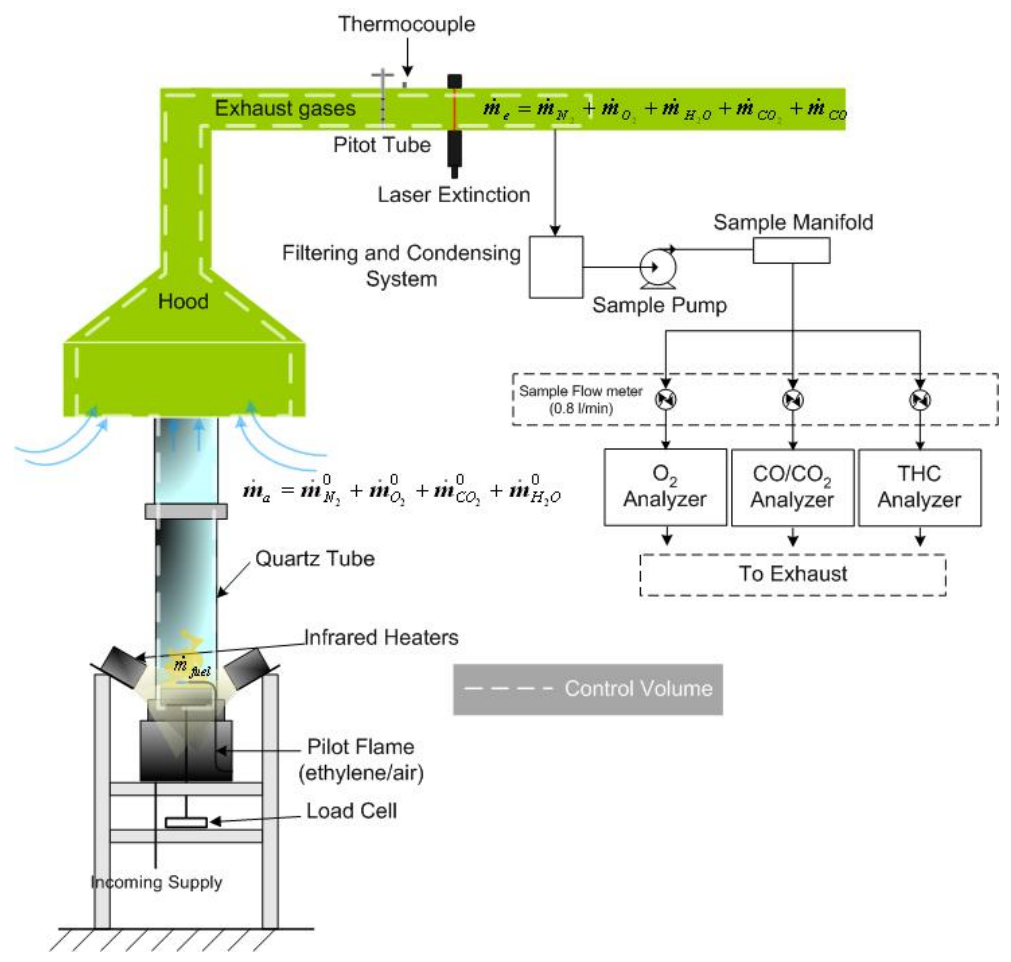

Figure 3: The Fire Propagation Apparatus 


\subsection{Fitting of the correlation}

A correlation is now fitted on experimental data, considering only experiments with a natural buoyant flow within the fuel layer (i.e. no forced flow enters the fuel sample). The FMC is here considered small and constant, hence its dependency is not considered relevant in this study.

Considering a closed basket, a flow and then a convective heat transfer can hardly develop within the fuel layer so that the ignition time scales as the particle reference time scales $t_{r e f, \mathrm{p}}$, showing only small dependencies on $C d L A I$ and $\Psi$ if the assumption is correct.

\begin{tabular}{|c||c|c|c|c|}
\hline$\Phi_{0}^{\prime \prime}\left[\mathrm{kW} \mathrm{m}^{-2}\right]$ & 8 & 12 & 15 & 25 \\
\hline \hline$B i[-]$ & 0.029 & 0.043 & 0.054 & 0.089 \\
\hline$t_{i g}[\mathrm{~s}]$ & 480 & 180 & 83 & 28 \\
\hline$t_{r e f, \mathrm{p}}[\mathrm{s}]$ & 842 & 373 & 238 & 85 \\
\hline$t_{r e f, \mathrm{~b}}[\mathrm{~s}]$ & $5.5310-2$ & $5.5310-2$ & $5.5310-2$ & $5.5310-2$ \\
\hline \hline$\Phi_{0}^{\prime \prime}\left[\mathrm{kW} \mathrm{m}{ }^{-2}\right]$ & 30 & 40 & 50 & \\
\hline \hline$B i[-]$ & 0.107 & 0.143 & 0.179 & \\
\hline$t_{i g}[\mathrm{~s}]$ & 15.5 & 10 & 8 & \\
\hline$t_{r e f, \mathrm{p}}[\mathrm{s}]$ & 60 & 32 & 20 & \\
\hline$t_{r e f, \mathrm{~b}}[\mathrm{~s}]$ & $5.5310-2$ & $5.5310-2$ & $5.5310-2$ & \\
\hline
\end{tabular}

Table 2: References and ignition times as a function of the incident flux for Pinus Halepensis litters

Therefore, the mean ratio of the ignition time on the reference particle time $\left(\overline{t_{i g}^{*}}\right)$ is calculated for every species and solid fractions. These results are presented in Table 3, proving that the ignition time scales roughly as an effusion time with a weak dependency on the fuel layer properties. Thus, the particle properties seem to drive the ignition process while the fuel layer properties (such as $C d L A I$ and $\Psi$ ) are accounting for an ignition time deviation regarding the effusion time. Other dimensionless groups are negligible when aiming at developing fire safety application since the discrepancies seem acceptable taking into account only the $C d L A I$ dependency in the ignition time correlation.

In the closed basket configuration, $\Psi$ is not expected to be a relevant parameter as explained earlier. However an increase of the in-depth radiation might affect the ignition process by increasing the temperature surrounding the solid particles, thus lowering the radiative and convective heat losses and then lowering ignition time. A correction for Eq. (1) that takes into account the in-depth radiation is then suggested introducing a $C d L A I$ dependency:

$$
t_{i g}^{*}=A(C d L A I)^{p}
$$

with $A=0.52$ and $p=-0.1$. The weak $C d L A I$ dependency exhibited by Eq. (18) seems to confirm the major role of the solid particles properties in configurations where no flow can develop within the fuel layer. This fitting 
is presented in Fig. 4, where the results have been re-written in the form of Fourier numbers with $F o_{i g}=\frac{\lambda_{s} \sigma_{s}^{2} t_{i g}}{4 \rho_{s} C p_{s}}$ being the experimental Fourier number and $F o_{r e f}=\frac{\lambda_{s} \sigma_{s}^{2} t_{r e f,}}{4 \rho_{s} C p_{s}} A(C d L A I)^{p}=\frac{\pi}{64 B i^{2}} A(C d L A I)^{p}$ being the reference Fourier number. A good agreement is found for $\mathrm{Fo}_{i g} \leq 50$ with a mean error of $7 \%$, demonstrating that the apparent thermally thick behaviour stands fairly for $F_{o i g} \leq 50$ corresponding to $B i \geq 0.08$. For $B i \leq 0.08$, other model [13], based on a thermally thin assumption, should be used. This experimental result agrees well with the analysis suggested on the theoretical thermal behaviour limit [12].

\begin{tabular}{|c||c|c|c|c|c|}
\hline Basket & PH 0.05 & PM 0.02 & PM 0.04 & PM 0.08 & PS 0.07 \\
\hline$C d L A I$ & 0.83 & 0.25 & 0.50 & 1.00 & 1.31 \\
\hline$\overline{t_{i g}^{*}}$ & 0.66 & 0.68 & 0.62 & 0.51 & 0.57 \\
\hline
\end{tabular}

Table 3: Mean ratio of ignition and effusion time for every species and solid fractions

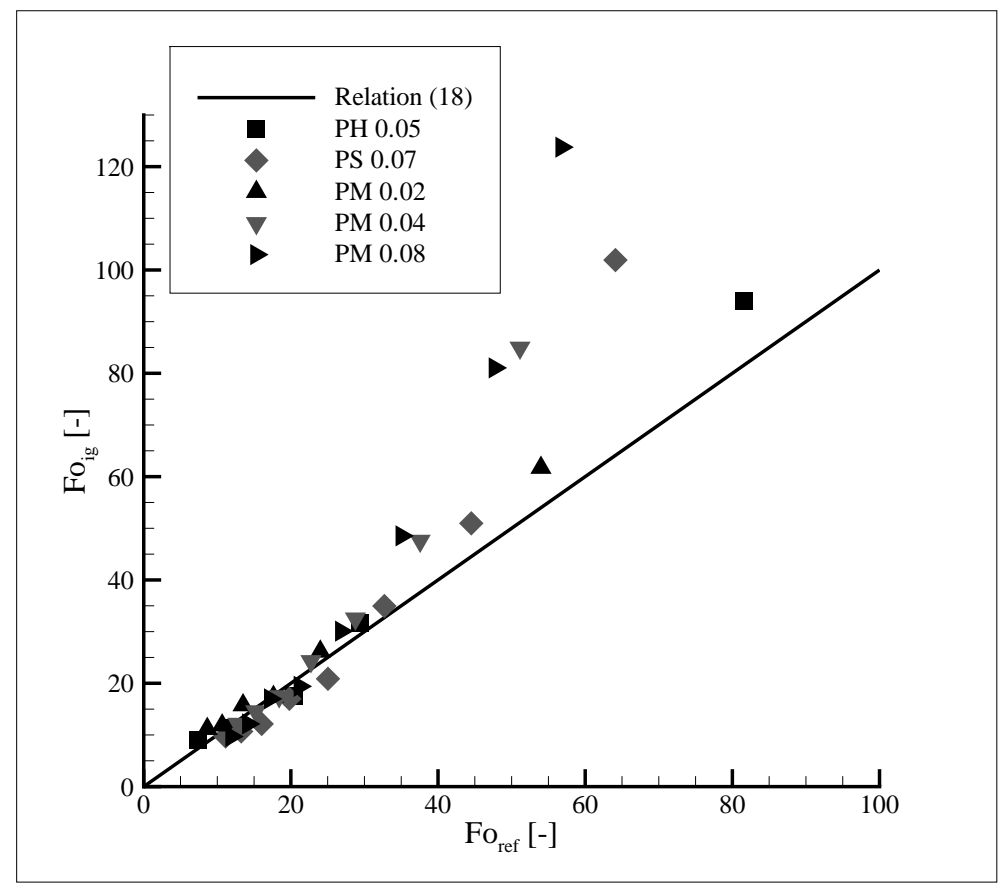

Figure 4: Eq. (18) and 0\% basket opening experimental data for different species and solid fraction 


\section{Concluding remarks}

In this study, a dimensional model for the ignition time of dry forest fuel layer has been developed. This model allows estimating the time to ignition of forest fuel layers when no flow is induced within the fuel layer and suggests some relevant dimensionless numbers when a natural buoyant flow can occur. In no induced-flow conditions, Eq. (18) can be used whereas another correlation that takes into account a $C d L A I$ and a $\Psi$ dependency should be developed when a flow is forced into the litter. The experimental comparison also demonstrated an apparent thermally thick behaviour of the solid particles during ignition process, which is in accordance with previous studies on that topic. In configurations where no flow can develop within the fuel layer, the ignition time has been found to depend priorly on the solid particles properties, scaling as the solid particle effusion time, with a weak dependency on the in-depth radiation phenomenon, causing a dependency on the dimensionless group $C d L A I$. In configurations where a natural buoyant flow can occur (caused by in-depth radiation and convective heat transfers), two dimensionless groups, $C d L A I$ and $\Psi$, have theoretically appeared to be relevant in the definition of the dimensionless time to ignition. Indeed, $C d L A I$ is responsible for both in-depth radiation and induced momentum effects and $\Psi$ stands for the hydrodynamic transitions within the fuel layer, which modify the convective heat transfer.

Several dimensionless numbers that influence the physics of litter ignition have also been enlighten, nevertheless their effect seem to be negligible when developing ignition time correlation with a typical margin of $10 \%$, which is acceptable for fire safety application. This simple and surprising result could be explained the following way: When looking at pine needle litter ignition problem, its dimensionless configuration space appears to be mainly extended in the $L A I$ direction. Indeed, relative variation of other dimensionless groups involved in this problem are practically small, even when considering numerous pine species and packing ratios, allowing development of a simple correlation. However, this result is specific to pine needle litters, hence the correlation should present other dependencies in order to be extended to other kind of highly porous media or other litters, such as leaf litters for instance.

\section{References}

[1] J. Torero, The SFPE Handbook of Fire Protection Engineering, fourth ed. National Fire Protection Association, 2008.

[2] P. Beaulieu and N. Dembsey, "Flammability characteristics at applied heat flux levels up to $200 \mathrm{kw} / \mathrm{m} 2$," Fire Mater., vol. 32:2, pp. 61-86, 2008 . 
[3] K. Saito, M. Delichatsios, S. Venkatesh, and R. Alpert, "Paper prepared for presentation at the fall technical meeting, eastern section," The Combustion Institute, 1988.

[4] F. Jiang, J. D. Ris, and M. Khan, "Absorption of thermal energy in PMMA by in-depth radiation," Fire Saf. J., vol. 44:1, pp. 106-112, 2009.

[5] N. Bal and G. Rein, "Numerical investigation of the ignition delay time of a translucent solid at high radiant heat fluxes," Combust. Flame, vol. 158, pp. 1109-1116, 2011.

[6] J. Torero and A. Simeoni, "Heat and mass transfer in fires: Scaling laws, ignition of solid fuels and application to forest fires," The Open Thermodynamics Journal, vol. 4, pp. 145-155, 2010.

[7] A. Simeoni, J. Thomas, P. Bartoli, P. Borowieck, P. Reska, F. Colella, P. Santoni, and J. Torero, "Flammability studies for wildland and wildland-urban interface fires applied to pine needles and solid polymers," Fire Saf. J., vol. 54, pp. 203-217, 2012.

[8] A. Lamorlette, A. Collin, and O. Séro-Guillaume, "Characterization of heat transfer between phases inside a porous medium as applied to vegetal set representations," Int. J. Heat Mass Transf., vol. 55, pp. 607$617,2012$.

[9] N. DeMestre, E. Catchpole, D. Anderson, and R. Rothermel, "Uniform propagation of a planar fire front without wind," Combust. Sci. and Tech., vol. 65, pp. 231-244, 1989.

[10] B. Monod, A. Collin, G. Parent, and P. Boulet, "Infrared radiative properties of vegetation involved in forest fires," Fire Saf. J., vol. 44, pp. 88-95, 2009.

[11] Z. Acem, A. Lamorlette, A. Collin, and P. Boulet, "Analytical determination and numerical computation of extinction coefficients for vegetation with given leaf distribution," Int. J. Thermal Sci., vol. 48, pp. 1501-1509, 2009.

[12] A. Lamorlette and F. Candelier, "Thermal behavior of solid particles at ignition: Theoretical limit between thermally thick and thin solids," Int. J.Heat Mass Transfer, vol. 82, pp. 117-122, 2015.

[13] P. Mindykowsky, A. Fuentes, J. Consalvi, and B. Porterie, "Piloted ignition of wildland fuels," Fire Saf. J., vol. 46, pp. 34-40, 2011.

[14] A. Grishin, A Mathematical Modelling of Forest Fires and New Methods of Fighting Them. Publishing House of the Tomsk University, Tomsk, Russia, 1997. 
[15] O. Séro-Guillaume and J. Margerit, "Modelling forest fires. part I: a complete set of equations derived by extended irreversible thermodynamics," Int. J.Heat Mass Transfer, vol. 45, pp. 1705-1722, 2002.

[16] D. Morvan, J. Dupuy, E. Rigolot, and J. Valette, "Firestar: a physically based model to study wildfire behaviour," Forest Ecol. Man, vol. 234S, p. S114, 2006.

[17] Y. Haseli, J. van Oijen, and L. de Goey, "Analytical solutions for prediction of the ignition time of wood particles based on a time and space integral method," Thermochim. Acta, vol. 548, pp. 65-75, 2012.

[18] C. Schemel, A. Simeoni, H. Biteau, J. Rivera, and J. Torero, "A calorimetric study of wildland fuels," Exp. Thermal Fluid Sci., vol. 32, pp. 1381-1389, 2008.

[19] D. Morvan, S. Méradji, and G. Accary, "Physical modelling of fire spread in grasslands," Fire Safety J., vol. 44, pp. 50-61, 2009.

[20] H. Nepf, "Drag, turbulence, and diffusion in flow trough emergent vegetation," Water Resour. Res., vol. 35, pp. 479-489, 1999.

[21] A. Lamorlette and A. Collin, "Analytical quantification of convective heat transfer inside vegetal structures," Int. J. Thermal Sci., vol. 57, pp. 78-84, 2012.

[22] J. Kaimal and J. Finnigan, Atmospheric boundary layer flows, their structure and measurement. Oxford University Press, 1994.

[23] F. Albini, "A model for fire spread in wildland fuels by radiation," Comb. Sci. and Technol., vol. 42, pp. 229-258, 1985.

[24] R. W. Fox, A. McDonald, and P. Pritchard, Introduction to Fluid Mechanics, sixth ed. Wiley, 2004.

[25] B. Benkoussas, J.-L. Consalvi, B. Porterie, N. Sardoy, and J.-C. Loraud, "Modelling thermal degradation of woody fuel particles," International Journal of Thermal Sciences, vol. 46, pp. 319-327, 2007.

[26] P. Bartoli, Forest fires: improvement of the knowledge of the coupling between flames and vegetative fuels. $\mathrm{PhD}$ thesis, Université de Corse, Pascal Paoli ; University of Edinburgh, 2011.

[27] V. Tihay, Contribution expérimentale et théorique pour la modélisation de la combustion dans les feux de forêts. PhD thesis, Université de Corse, Pascal Paoli, 2007. 
[28] Z. Acem, G. Parent, B. Monod, G. Jeandel, and P. Boulet, "Experimental study in the infrared of the radiative properties of pine needles," Exp. Therm. Fluid Sci., vol. 34, pp. 893-899, 2010.

[29] "Deliverable D-02-02-A: Physical, chemical and thermal characteristics of the wildland fuel particles - answer from the partners," EUFIRELAB: A Wall-Less Laboratory for Wildland Fire Sciences and Technologies in the Euro-Mediterranean Region, vol. EU FP5 Program EVR1-CT-200240028, 2004.

[30] M. Chaos, "Spectral aspects of bench-scale flammability testing: Application to hardwood pyrolysis," in Fire Safety Science - Draft proceeding of the eleventh International Symposium, 2014.

[31] N. Cheney, J. Gould, and W. Catchpole, "The influence of fuel, weather and fire shape variables on fire-spread in grasslands," Int. J. Wildland Fire, vol. 3(1), pp. 31-44, 1993.

[32] M. Ghisalberti and H. Nepf, "The structure of the shear layer in flows over rigid and flexible canopies," Environmental Fluid Mechanics, vol. 6, pp. 277-301, 2006.

[33] C. Williamson, "The natural and forced formation of spot-like "vortex dislocations" in the transition of a wake," J. Fluid Mech., vol. 243, pp. 393-441, 1992. 